\title{
Cornelia de Lange Syndrome: NIPBL haploinsufficiency downregulates canonical Wnt pathway in zebrafish embryos and patients fibroblasts
}

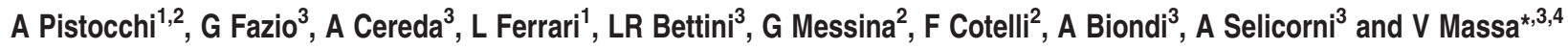

Cornelia de Lange Syndrome is a severe genetic disorder characterized by malformations affecting multiple systems, with a common feature of severe mental retardation. Genetic variants within four genes (NIPBL (Nipped-B-like), SMC1A, SMC3, and HDAC8) are believed to be responsible for the majority of cases; all these genes encode proteins that are part of the 'cohesin complex'. Cohesins exhibit two temporally separated major roles in cells: one controlling the cell cycle and the other involved in regulating the gene expression. The present study focuses on the role of the zebrafish nipblb paralog during neural development, examining its expression in the central nervous system, and analyzing the consequences of nipblb loss of function. Neural development was impaired by the knockdown of nipblb in zebrafish. nipblb-loss-of-function embryos presented with increased apoptosis in the developing neural tissues, downregulation of canonical Wnt pathway genes, and subsequent decreased Cyclin D1 (Ccnd1) levels. Importantly, the same pattern of canonical WNT pathway and CCND1 downregulation was observed in NIPBL-mutated patient-specific fibroblasts. Finally, chemical activation of the pathway in nipblb-loss-of-function embryos rescued the adverse phenotype and restored the physiological levels of cell death.

Cell Death and Disease (2013) 4, e866; doi:10.1038/cddis.2013.371; published online 17 October 2013

Subject Category: Neuroscience

Cornelia de Lange Syndrome (CdLS) is a multiple malformation syndrome typically involving developmental delay, specific facial features, behavioral abnormalities, and major congenital malformations. Almost any organ can be affected, but the most commonly observed defects are the ones that affect the neurodevelopmental, gastrointestinal, and musculoskeletal systems. There is a broad spectrum of clinical involvement, with increasing recognition of a much milder phenotype than previously appreciated. Clinically, a common feature of CdLS is mental retardation that is often associated with enlarged brain ventricles, thinning or atrophy of white matter, brainstem hypoplasia, and cerebellar vermal hypoplasia or agenesis. ${ }^{1-3}$ During development, the central nervous system (CNS) arises from the neural plate that folds up on its anterior-posterior (AP) axis to form a tube divided in three main units: the anlagen of the prosencephalon (forebrain), the mesencephalon (midbrain), and the rhombencephalon (hindbrain). Caudally, the tube will develop into the spinal cord. These coordinated events are achieved through a finely tuned balance between cell proliferation and programmed cell death. Within each region, a large diversity of neuronal cell types is generated, each with distinct identities in terms of their morphology, axonal trajectory, synaptic specificities, and neurotransmitters. ${ }^{4}$ This fundamental specification is controlled by dorsoventral (DV) and AP gradients of molecules. For example, components of the Wnt family are known to induce anterior structures (such as forebrain), whereas bone morphogenetic proteins are responsible for neurogenesis in the dorsal neural tube. ${ }^{5}$

Genetically, CdLS is caused by both autosomal-dominant and X-linked mutations. Approximately, $60 \%$ of the cases are due to genetic defects in one of the four genes: NIPBL (Nipped-B-like), SMC1A, SMC3, and the recently identified HDAC 8. ${ }^{1,6}$ NIPBL gene was the first to be found associated with CdLS. SMC1A was subsequently identified as an additional gene whose disruption produces the phenotypic findings of the CdLS, whereas one CdLS case was reported having a mutation in SMC3. HDAC8 was found to be mutated in CdLS patients, consistent with its role in controlling the acetylation status of SMC3. Most recently, mutations in RAD21, a structural protein in the cohesin complex, have been found in children with a spectrum of disabilities overlapping with the CdLS syndrome, presenting with milder cognitive impairments consistent with a so-called

\footnotetext{
${ }^{1}$ Department of Medical Biotechnologies and Translational Medicine, University of Milan, Via Viotti, 3/5, 20133, Milan, Italy; ${ }^{2}$ Department of Biosciences, University of Milan, Via Celoria, 26, 20133, Milan, Italy and ${ }^{3}$ Department of Paediatrics, S.Gerardo Hospital/Fondazione MBBM, University of Milano-Bicocca, Via Pergolesi, 33, 20900, Monza, Italy

${ }^{*}$ Corresponding author: V Massa, Department of Paediatrics, S.Gerardo Hospital/Fondazione MBBM, University of Milano-Bicocca, Via Pergolesi, 33, 20900, Monza, Italy. Tel: + 39039 2333661; Fax: + 39039 2332167; E-mail: v.massa@ @sgerardo.org

${ }^{4}$ Present address: Department of Health Sciences, University of Milan, Via A. Di Rudiní, 8, 20142, Milan, Italy

Keywords: Cornelia de Lange Syndrome; cyclins; zebrafish; fibroblasts; NIPBL; apoptosis

Abbreviations: CdLS, Cornelia de Lange Syndrome; Ccnd1, Cyclin D1; Ccnd2, Cyclin D2; CNS, central nervous system; AP, anterior-posterior; DV, dorsoventral; WISH, whole-mount in situ hybridization; RPMI, Roswell Park Memorial Institute serum; q-PCR, quantitative RT-PCR; TBST, tris-buffered saline/Tween 20 buffer; ABC, active form of beta-catenin; Fmi/Stan, Flamingo/Starry

Received 31.7.13; accepted 01.8.13; Edited by G Raschellá
} 

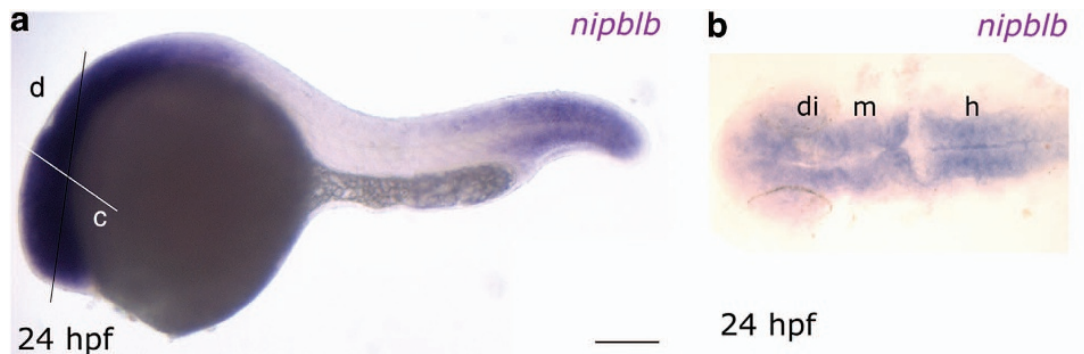

$24 \mathrm{hpf}$
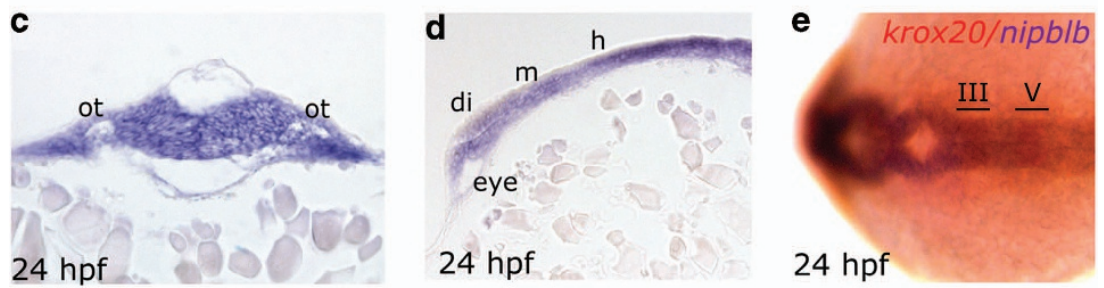

Figure 1 Expression analysis of nipblb. (a-d) WISH with nipblb-specific probe at $24 \mathrm{hpf}$. (a) Whole-mount embryo showing modulated nipblb expression along the AP axis. $\mathbf{c}$ and $\mathbf{d}$ indicate the position of histological sections reported in $\mathbf{c}$ and $\mathbf{d}$, respectively. (b) Dorsal view (anterior to the left) of the different regions of nipblb expression in the CNS. (c) Transverse section of the neural tube showing that the nipblb transcript is ubiquitously expressed along the DV axis. (d) Longitudinal section showing nipblb expression along the AP axis. (e) Comparison between nipblb (in blue) and krox20 (in red) expressions showing that nipblb extends posteriorly as far as rhombomere $\mathrm{V}$ at $24 \mathrm{hpf}$. di, diencephalon; m, midbrain; h, hindbrain; ot, otocystis. Scale bar, $100 \mu \mathrm{m}$

coesinopathy. ${ }^{3}$ All these genes encode proteins involved in the 'cohesin complex'.

The cohesin complex is evolutionarily conserved from fungi to human cells, and its canonical role is the regulation of chromatid separation during cell division. ${ }^{7}$ In particular, the cohesin complex controls sister chromatid cohesion during $S$ phase. In the somatic cells of vertebrates, four core subunits forming the complex have been identified: SMC1 (SMC1A or SMC1B), SMC3, SCC1, and either SA1 or SA2. Associated with the core complex, other proteins assist during cell division, for example, NIPBL, which appears to facilitate the uploading of the complex, or WAPL, which enables complex dissociation. ${ }^{8}$

A non-canonical role has recently been observed, whereby the cohesin complex also represents a key regulator of gene expression. In Drosophila, NIPBL ortholog (i.e., Nipped-B) has been shown to participate in the long-distance activation of cut and Ultrabithorax gene expression. ${ }^{9}$ In zebrafish (Danio rerio), cohesins regulate runx transcription factors and hematopoiesis. ${ }^{10}$ Interestingly, it seems that functionally, the cell cycle control and gene expression regulation are temporally separated. For example, Drosophila mutants for orthologs of cohesin complex genes show a defect in axon retraction that physiologically occurs in post-mitotic neurons, hence in cells that have exited the cell cycle. ${ }^{11}$ In zebrafish, cohesins are expressed in both proliferating and non-proliferating cells, ${ }^{12}$ while mice that are heterozygous for Nipbl have severe developmental defects and generally altered gene expression in the absence of cell cycle or sister chromatid impairment. $^{13}$

A nipbl-deficiency model in zebrafish shows altered expression of genes involved in endodermal differentiation along with a spectrum of heart, gut, and visceral organ defects that bear a striking similarity to those observed in CdLS patients. ${ }^{14}$ In the present study, we have used two combined approaches, zebrafish embryos and patient-derived cells, in order to dissect molecular mechanisms underlying the neurodevelopmental disorders. First, the effects of nipblb loss of function in the developing CNS of zebrafish, in particular at the hindbrain level, were characterized. The results obtained from functional studies in zebrafish embryos were then confirmed in fibroblasts of CdLS patients with known mutation in NIPBL.

\section{Results}

Zebrafish nipblb is expressed in the CNS. In accordance with the literature, ${ }^{12,14}$ nipblb was ubiquitously expressed during early somitogenesis in zebrafish embryos. The expression progressively decreased in the trunk and appeared specifically localized in the CNS at $24 \mathrm{hpf}$ (hours post fertilization) (Figure 1a). As seen in this dorsal view, expression of nipblb (Figure 1b) was detected in the diencephalon and mesencephalon, and highlighted the progression of hindbrain ventricle opening. Histological longitudinal and transverse sections of $24 \mathrm{hpf}$ embryos clearly showed that nipblb is expressed throughout the entire dorsal-ventral (DV) and AP axis of the CNS (Figures 1c and d). Colocalization with krox20, a marker of rhombomeres III and $\mathrm{V}^{15}$ showed that nipblb expression extended caudally as far as rhombomere $\mathrm{V}$ at this developmental stage (Figure 1e).

Inhibition of Nipblb function in zebrafish embryos affect CNS development. Embryos injected with nipblb-morpholino (MO) began to exhibit defects at $24 \mathrm{hpf}$, including brain defects ( $50 \%$ of nipblb-MO injected, total $n=200$, Figures $2 \mathrm{a}$ and b). In particular, nipblb-loss-of-function embryos were microphthalmic and microcephalic. In addition, nipblbMO-injected embryos were presented with a short, curved tail ( $40 \%$ of nipblb-MO injected, total $n=180$, Figure $2 b$ ), a phenotype previously described by Muto et al. ${ }^{14}$ following nipblb loss of function. nipblb-MO efficiency and specificity 

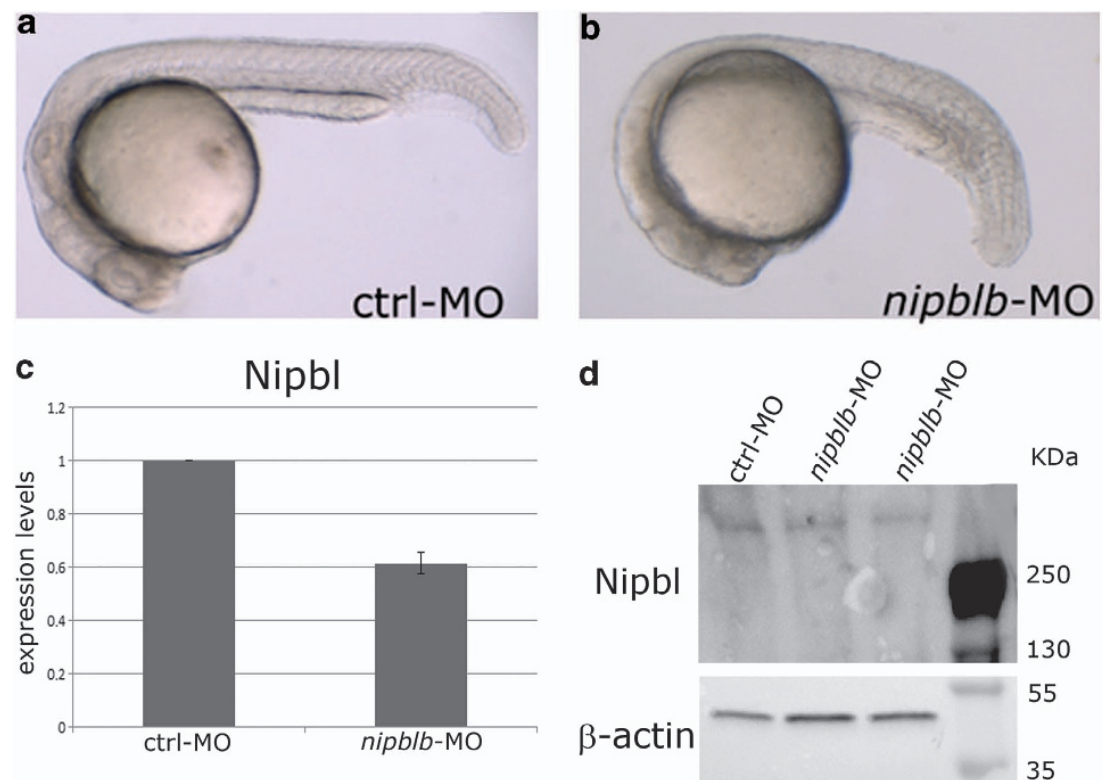

Figure 2 MO knockdown of nipblb. (a and b) Morphology of living embryos at 24 hpf. (a) Embryos injected with the ctrl-MO. (b) Embryos injected with the nipblb-MO presenting brain defects and a curved tail. (c) Nipbl is reduced in MO-injected embryos by $39 \pm 0.04 \%$ compared with controls (expressed as mean of triplicate densitometric analyses normalized against controls). (d) Western blot of protein extracted from minimum 30 embryos per experimental group showing reduced levels of Nipbl (316 KDa) in two different batches of $24 \mathrm{hpf}$ nipblb-MO-injected embryos compared with controls at the same developmental stage. Lower band is beta-actin ( $50 \mathrm{KDa})$

were verified by western blot experiments in which Nipbl protein levels were reduced by $39 \%$ in nipblb-MO-injected embryos at $24 \mathrm{hpf}$, compared with stage-matched control embryos (Figures 2c and d).

nipblb downregulation affects cell survival but not cell proliferation. When compared with controls, nipblb-MOinjected embryos showed increased programmed cell death in the CNS at $24 \mathrm{hpf}$, whereas apoptosis was less pronounced in CNS of control embryos (Figures 3a-d). Interestingly, the TUNEL staining showed that the apoptotic cells were most evident in the tissues expressing the nipblb gene (compare Figure $1 \mathrm{a}$ and Figures $3 \mathrm{~b}-\mathrm{d}$ ). Previous studies in zebrafish described p53-dependent apoptosis following cohesin smc3 knockdown, and p53 upregulation in mutants for the cohesin subunit rad21. ${ }^{16,17}$ It is well known that MO molecules could elicit undesirable off-target effects such as activation of the p53 protein. Importantly, reversal of p53-dependent cell death by p53 knockdown did not affect cell death caused by specific gene loss of function. ${ }^{18}$ To verify that apoptosis in nipblb-MO-injected embryos was due to the specific loss of function of this gene and not a result of p53 activation, we co-injected p53-MO with nipblb-MO. In p53-MO + nipblb-MO-injected embryos, the amount of apoptotic cells were comparable to nipblb-loss-of-function embryos, which verified that neural apoptosis was specifically caused by knocking down nipblb $(n=42)$ (Supplementary Figure S1). These results suggest that nipblb is required for cell survival during neurulation in zebrafish embryos.

To determine whether nipblb loss of function also alters the proliferation rate, embryos were stained with the proliferative cell nuclear antigen antibody. As expected from previously published studies on cohesin loss of function, ${ }^{10,19}$ we found that entry into the $S$ phase was not compromised, as the number of proliferative cell nuclear antigen-positive cells in nipblb-MO-injected embryos at $24 \mathrm{hpf}$ was not significantly increased in comparison with controls (Supplementary Figure S2).

nipblb loss of function alters Wnt signaling pathway expression. Owing to the expression of nipblb throughout the CNS, we further investigated whether CNS AP formation was altered in nipblb-loss-of-function embryos at $24 \mathrm{hpf}$. The expression of AP identity markers, such as hoxb2a, ${ }^{20}$ pax2a, ${ }^{21}$ and $k r o x 20,{ }^{15}$ was not altered in nipblb-MO-injected embryos (Supplementary Figure S3), suggesting that the CNS is patterned correctly. However, we found that wnt1 expression $^{22}$ was severely altered in the hindbrain of nipblbMO-injected embryos at $24 \mathrm{hpf}$ ( $80 \%$, total analyzed embryos $n=120$; Figures $4 \mathrm{a}$ and b). It was possible to observe nipblb knockdown phenotypes with different degrees of fusion of the wnt 1 signal in the hindbrain between rhombomere II and the spinal cord: the most severe phenotype included a complete fusion of the wnt 1 hindbrain signals ( $20 \%$, total $n=120)$, the intermediate phenotype expressed multiple points of wnt1 fusion along the AP hindbrain axis (40\%, total $n=120)$, and the least severe phenotype presented either as a single point of wnt 1 fusion or as an incomplete opening of the ventricle (20\%, total $n=120$, Figures $4 \mathrm{a}$ and $\mathrm{b}$ ).

To investigate whether the observed defects were specific for the mis-regulation of wnt1 expression following nipblb loss of function or were caused by morphological defects in hindbrain formation, we analyzed the expression of atoh1, a marker of hindbrain dorsal progenitors and ventricle opening. ${ }^{23}$ atoh 1 expression was not altered in nipblb-MO-injected embryos at $24 \mathrm{hpf}(90 \%$, total $n=50$; Supplementary Figures S4A and B), showing that anatomical patterning of the developing brain is not affected by nipblb loss of function. 

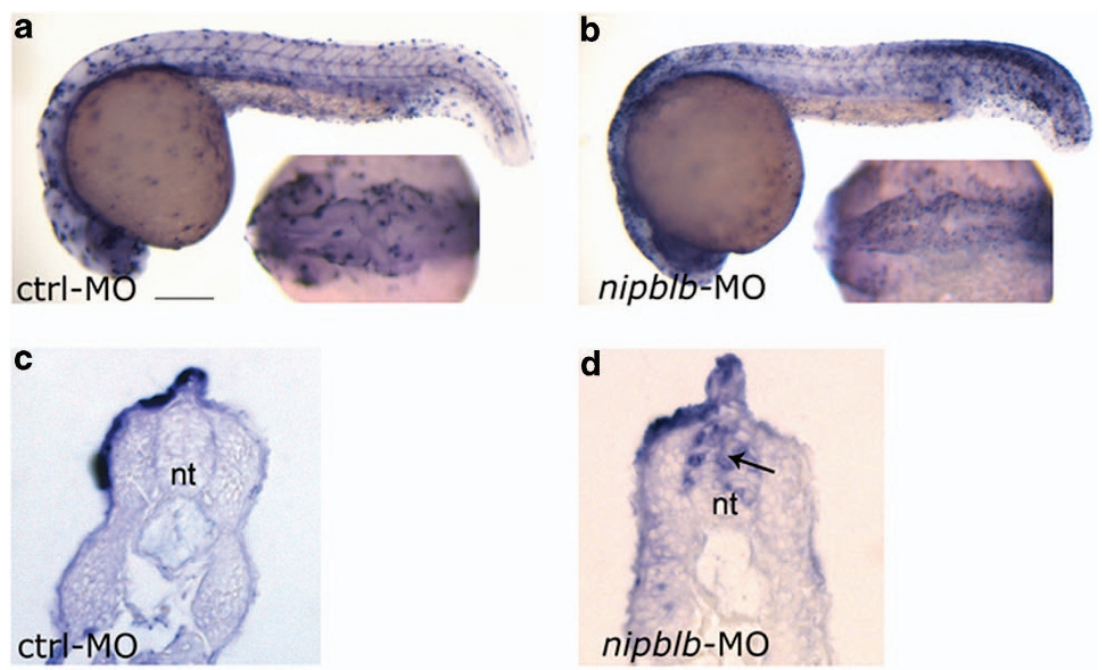

Figure 3 Apoptosis is increased in the CNS of nipblb-loss-of-function embryos. (a and $\mathbf{b}$ ) Apoptotic cells labeled with TUNEL were strongly increased in (b) nipblb-MOinjected embryos at $24 \mathrm{hpf}$ in comparison with control embryos (a) at the same developmental stage. (c and d) Transverse sections indicated prevalent cell death in the neural tube of (d, arrow) nipblb-MO-injected embryos in comparison with controls (c). nt, neural tube. Scale bar, $100 \mu \mathrm{m}$
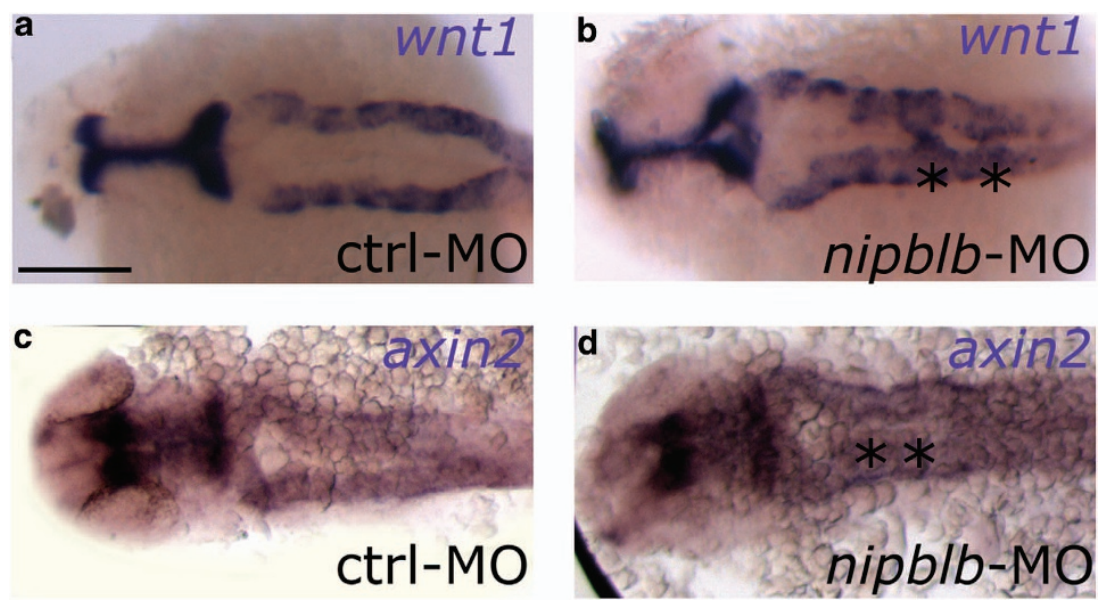

Figure 4 wnt1 expression is specifically mis-regulated in the hindbrain of nipblb-MO-injected embryos. (a and $\mathbf{b})$ wnt1 expression in controls and nipblb-MO-injected embryos at $24 \mathrm{hpf}$. Altered wnt1 expression in the hindbrain of (b) nipblb-MO-injected embryos at $24 \mathrm{hpf}$ in comparison with (a) controls at the same developmental stage. Asterisks indicate multiple points of wnt 1 fusion along the AP hindbrain axis of (b) nipblb-MO-injected embryos. (c and $\mathbf{d}$ ) axin2 expression showing comparable defects of patterning in nipblb-MO-injected embryos (d) in comparison with controls (c). Scale bar, $100 \mu \mathrm{m}$

As defects in neural tube patterning might disrupt neuronal differentiation, we examined the dopaminergic population (thyrosin-hydroxilase-positive neurons) at $24 \mathrm{hpf}$ following nipblb loss of function. It is well known that formation of mesencephalic dopaminergic neurons is directed not only by diffusable signals from the notochord, floor plate, and isthmic organizer such as Shh and Fgf, but also by Wnt 1 and other extrinsic factors. ${ }^{24}$ In nipblb-loss-offunction embryos, we found that neurodifferentiation of this population was not adversely affected (Supplementary Figures S4C and D).

Importantly, comparable alterations in gene distribution observed in wnt1 were also observed in axin2 expression pattern, ${ }^{25}$ a key downstream component of the pathway typically used as a readout of the cascade (Figures $4 \mathrm{c}$ and d).
Expression profiling of the WNT/beta-catenin pathway target genes in patients fibroblasts and nipblb-MO-injected embryos. In zebrafish embryos, nipblb loss of function resulted in alterations of the Wnt/beta-catenin pathway, modeling the molecular defects underlying NIPBLmutated CdLS patients. Hence, we sought to analyze the expression profile of this signaling pathway in fibroblasts of patients, comparing it with that of cells obtained from age-, ethnicity- and gender-matched healthy controls. Fibroblasts are believed to represent a valid model for cohesin deficiency in humans ${ }^{6}$ as in studies on gene expression, consistent altered profiles were observed in primary cultures of fibroblasts and lymphoblastoid cell lines. ${ }^{6}$

It was possible to exclude involvement of the non-canonical pathway, as both $D V L 1$ and $D V L 2^{26}$ are comparable in patients and control group (Supplementary Figures S5A and B). 
a

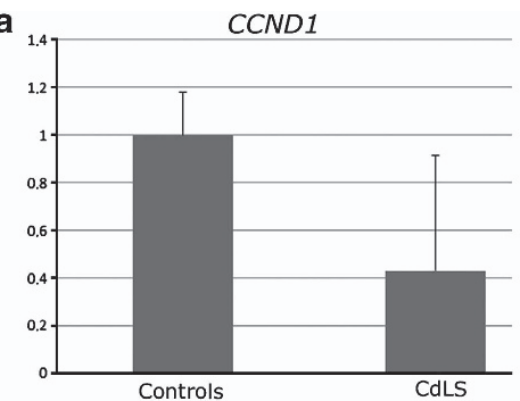

C
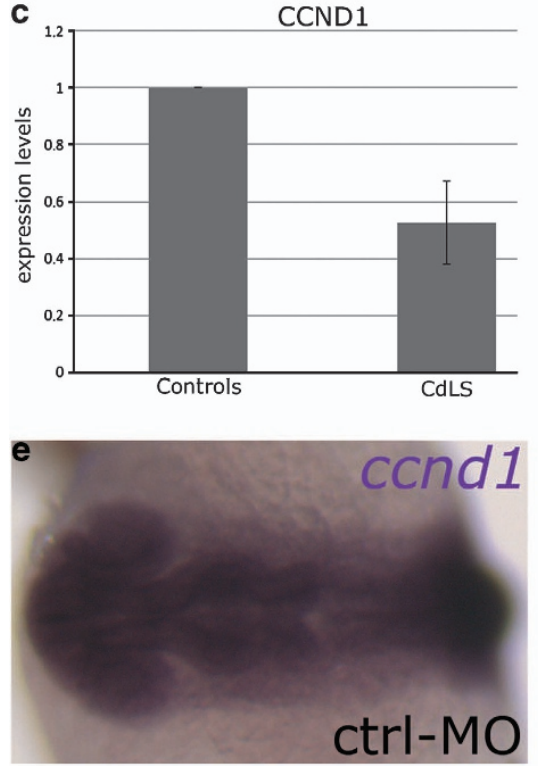

g

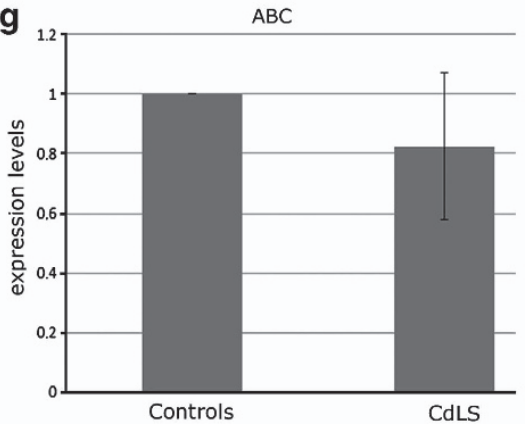

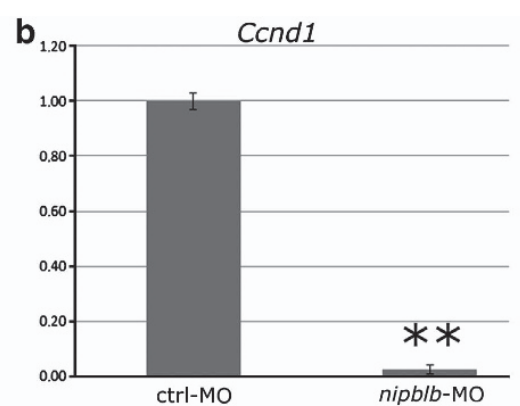
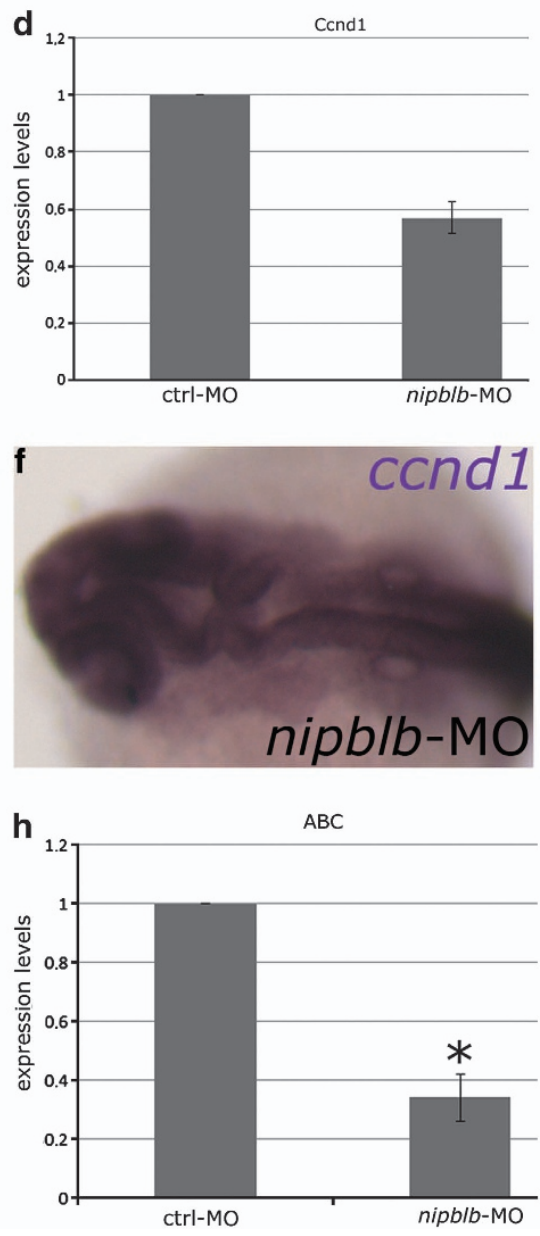

Figure 5 The canonical Wnt pathway is downregulated in animal and human models of CdLS. (a and $\mathbf{b})$ Human (CCND1) and zebrafish (ccnd1) real-time analysis in (a) patients-specific fibroblasts and in (b) nipblb-MO-injected embryos showing a reduction trend of the transcripts in both models (asterisks in $\mathbf{b}$ represent $P<0.01$ ). (c and $\mathbf{d}$; $\mathbf{g}$ and $\mathbf{h}$ ) Western blot analyses. Quantification of relative protein expression after normalization on beta-actin. The quantification was done on three (zebrafish) or two (human) independent experiments and the data are expressed as fold change over relative controls with standard errors. (c and d). The reduction of CCND1 is confirmed at the protein levels in CdLS patient-derived cells (c) and in nipblb-MO-injected embryos (d). (e and f) WISH analysis showing decreased expression levels of ccnd1 in nipblb-MO-injected embryos (f) compared with control embryos (e) at the same developmental stage. ( $\mathbf{g}$ and $\mathbf{h}$ ) Levels of ABC were reduced in both CdLS models, that is, human ( $\mathbf{g}$ ) and zebrafish (h), compared with specific controls. Asterisk in $\mathbf{h}$ represents $P<0.05$. (e and $\mathbf{f})$ Dorsal view, anterior to the left

Analysis of the expression of direct targets of the canonical pathway, such as Cyclins, by quantitative PCR (q-PCR) showed a trend toward the reduction of Cyclin D1 (CCND1) in NIPBL-mutated patients compared with healthy controls (Figure 5a). As it is known that Cyclins can compensate for the deficiency of other isoforms in mammals, ${ }^{27}$ we also analyzed Cyclin D2 (CCND2) and found it to be significantly upregulated $(P<0.01)$ (Supplementary Figure S5C).
Consistently, a mean reduction of CCND1 levels of almost $50 \%$ was observed in patients samples when compared with healthy controls (Figure 5c, Supplementary Figure 5D). Ccnd1 was also found to be significantly downregulated in nipblb-MO-injected embryos by q-PCR (Figure 5b), western blot analysis with a decrease of Ccnd1 expression by $42 \%$ (Figure 5d), and whole-mount in situ hybridization (WISH) analyses (Figures $5 e$ and $\mathrm{f}$ ). The latter showed that the 

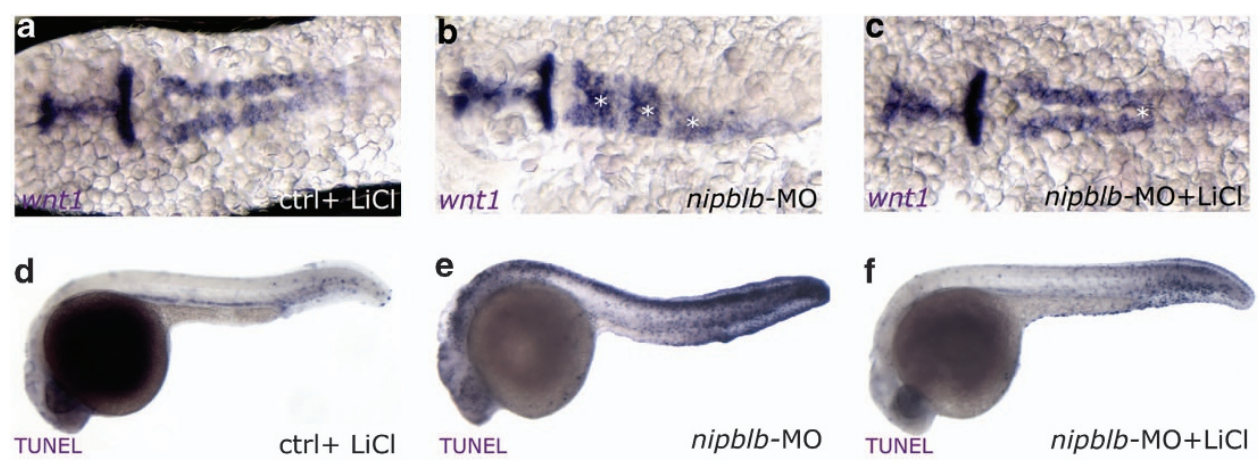

h

Cond 1
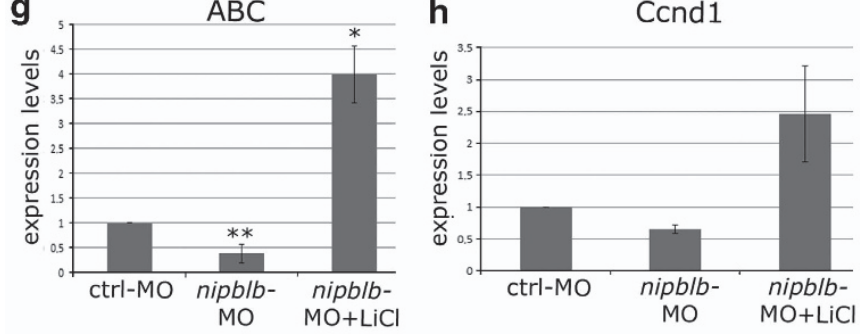

i
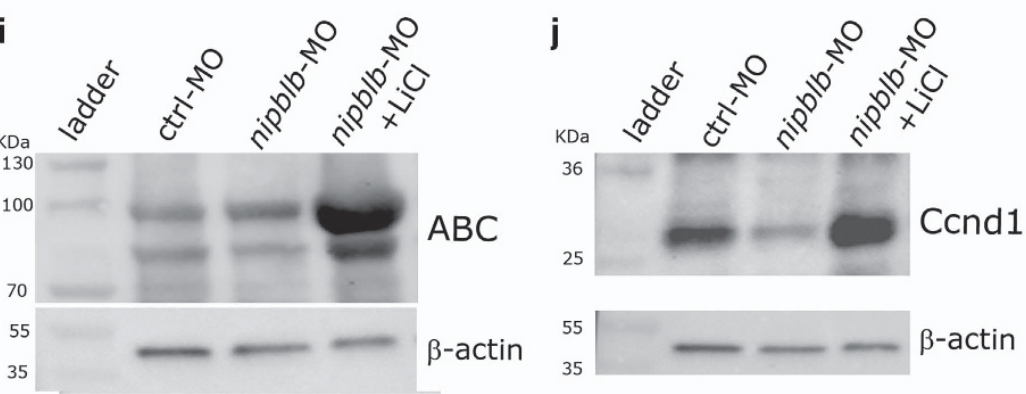

Figure 6 Molecular and morphological defects in CdLS zebrafish model can be rescued by LiCl treatment. (a-c) Dorsal view (anterior on the left) of flat-mounted embryos showing altered wnt1 expression in nipblb-MO-injected embryos (b) with multiple points of fusion (asterisks) in comparison with controls (a), and restored wnt1 expression to normal pattering in nipblb-MO-injected embryos treated with LiCl (c). (d and f) Lateral view of whole embryos stained with TUNEL assay showing that treatment with LiCl (f) rescues the morphological cephalic defects and reduces level of abnormal apoptosis (e) restoring physiological levels comparable to control embryos (d). ( $\mathbf{g}-\mathbf{j})$ Western blot analyses showing that $\mathrm{ABC}(\mathbf{g}-\mathbf{i})$ and $\mathrm{Ccnd1}(\mathbf{h}-\mathbf{j})$ protein levels are strongly increased in nipblb-MO-injected LiCl-treated embryos in comparison with nipblb-MO-injected embryos. Western blot quantification was done on three (zebrafish) independent experiments, and the data are expressed as fold change in nipblb-MO-injected embryos and nipblb-MO-injected LiCl-treated embryos over controls with standard errors. Asterisks in $\mathrm{g}$ represent ${ }^{*} P<0.05$ or ${ }^{* *} P<0.01$

expression of ccnd1 was altered in nipblb-MO-injected embryos at $24 \mathrm{hpf}$ in comparison with controls $(40 \%$, total $n=30)$.

In addition, we found an average of $18 \%$ reduction of the active form of beta-catenin $(A B C)^{28}$ in patients compared with controls after normalization (Figure $5 \mathrm{~g}$, Supplementary Figure 5D). The same pattern was observed in nipblb-MOinjected embryos with a statistically significant reduction of $A B C$ levels of $80 \%$ (Figure $5 \mathrm{~h}$ ).

Rescue of nipblb deficiency phenotype by chemical induction of canonical Wnt pathway. As our data suggested that the adverse phenotype caused by knocking down of nipblb in zebrafish embryos and in patients fibroblasts was mediated by attenuation of the canonical Wnt pathway with a subsequent decreased in Ccnd1 expression, we sought to rescue the phenotype in nipblb-loss-of-function embryos by chemical induction of the Wnt pathway by lithium chloride (LiCl) treatment. ${ }^{29}$ The concentration used in three sets of experiments failed to produce visible effects in control embryos (minimum 30 per experiment), but was able to strikingly reverse the defects induced by nipblb-MO injection (minimum 30 per experiment). In particular, the cephalic structures showed a restored morphology, and the wnt1 distribution pattern is similar to that of control embryos (Figures 6a-c). Apoptosis levels determined by TUNEL staining were reduced after $\mathrm{LiCl}$ treatment in nipblb-MOinjected embryos to levels comparable to those of control embryos (Figures 6d-f). Western blot analysis of nipblb-MOinjected embryos treated with $\mathrm{LiCl}$ showed that the canonical Wnt pathway was induced, as ABC levels were increased by fourfold compared with controls and more than 10-fold compared with nipblb-MO-injected embryos (Figures 6g-i). Both differences were statistically significant. Notably, Ccnd1 protein levels following $\mathrm{LiCl}$ treatment were induced more than 3.7-fold compared with nipblb-MO-injected embryos and 2.4-fold increase compared with controls. (Figures 6h-j). 


\section{Discussion}

In this study, two different modeling strategies were used to analyze abnormalities underlying CdLS, focusing on NIPBL, which is the most commonly mutated gene in patients affected by the disease. ${ }^{1,30} \mathrm{~A}$ developmental study in vivo using zebrafish embryos was initially used to identify gene pathways that might contribute to the phenotype, followed by a validation study in which primary cultures of CdLS patients fibroblasts were subsequently tested.

First, the expression of nipblb in the CNS of the zebrafish embryos at $24 \mathrm{hpf}$, the time when the neurulation process is almost completed, was evaluated. This alleviates an existing data gap in the literature, as the expression pattern of zebrafish nipblb at different developmental stages had been previously reported. ${ }^{12,14}$ The results showed a dynamic pattern of expression regulated spatio-temporally, with specific localization at the level of the cephalic neural tissues. The other zebrafish paralog nipbla had been reported with a similar expression pattern and function in the developing visceral organs. ${ }^{14}$

Using MO technology, a model of haploinsufficiency in zebrafish embryos was generated. This model mimics the genetic haploinsufficiency of CdLS patients with mutation in NIPBL. The nipblb-loss-of-function embryos showed severe alterations of the head morphology, with various degrees of microcephaly, microphthalmia, and incomplete development of these cephalic structures.

This adverse phenotype correlated with increased apoptosis in the areas where nipblb is normally expressed, suggesting that the cohesins have an important functional role in cell survival of specific tissues during vertebrate development. In our system, the proliferation rate seemed unaffected. Interestingly, the expression distribution of a number of markers of AP development appeared comparable to control embryos. wnt1 distribution, on the other hand, was significantly impaired at the hindbrain level, the prospect pons, medulla oblongata, and cerebellum, in mammals. We ruled out that wnt 1 expression alterations were caused by abnormalities in anatomy of developing structures as atoh1, which expression covers the entire dorsal and lateral aspect of the cerebellar anlage, was properly distributed in nipblbMO-injected embryos. ${ }^{31,32}$ The wnt 1 localization, however, failed to be restricted to the lateral tissues in these embryos. This is consistent with previous data indicating the presence of underlying anterio-posterior pattern in the absence of Wnt signals. ${ }^{33-35}$

The canonical Wnt pathway was found to be altered in nipblb-MO-injected embryos and in patients fibroblasts. In both cases, the ABC was downregulated. Ccnd1, a known direct target of the pathway, was consequently downregulated in both models. Noticeably, the abnormal cephalic phenotype observed in nipblb-MO-injected embryos is strongly reminiscent of that observed after Cond1 knockdown.

Using $\mathrm{LiCl}$ as a well-known chemical activator of the Wnt pathway, ${ }^{34,36}$ which negatively regulates GSK3 $\beta$ inhibition of beta-catenin, ${ }^{37}$ it was possible to rescue the adverse phenotype in nipblb-MO-injected embryos at all levels: anatomical cephalic structures appeared morphologically normal, the programmed cell death levels were restored to physiological levels seen in controls, and Ccnd1 protein levels were increased. Interestingly, wnt1 pattern of expression appeared rescued in zebrafish nipblb-MO-injected embryos by $\mathrm{LiCl}$ treatment, suggesting that in our model, the primary adverse event is at the Gsk3 $\beta$-Beta-catenin complex level, and that chemical compensation triggers a negative feedback on wnt1 expression control. In fact, it has been reported a negative feedback through upregulation of Axin2 in a cancer model. ${ }^{38}$

Our CdLS zebrafish model suggests a role for the canonical Wnt pathway perturbation in CNS abnormalities. In a murine model of autosomic recessive microcephaly, the brain alterations are caused by decrement in expression levels of Wnt1-regulated genes, and overexpression of beta-catenin in neural cells could rescue the defects. ${ }^{39}$ Several animal models with alterations in Wnt-related gene expression show partially overlapping phenotype: the well-known masterblind ( $m b /)$ mutant, which develops reduction of the telencephalon, optic vesicles ,and an abnormal AP patterning, was found to be a spontaneous axin 1 mutant. ${ }^{34,35}$ Since then several mouse (for example, Hesx1, Six3, and Tcf3), ${ }^{40-42}$ and zebrafish mutants ${ }^{43,44}$ have been described with comparable phenotype at the AP patterning of the CNS during embryonic development. In humans, HESX1 and SIX3 have been found causative of hypopituitarism and septo-optic dysplasia, ${ }^{45,46}$ a developmental disorder characterized by severe defects in the brain and eye. In 1996, a case report described a 5-year-old CdLS patient autopsy that revealed hypoplasia of the optic systems, hypothalamic nuclei, corpus callosum, and cerebellar vermis. Moreover, the septum pellucidum, fornix, and anterior commissure were rudimentary. This case showed features of septo-optic dysplasia combined with commissural dysplasia and cerebellar vermian hypoplasia. ${ }^{47}$

It is known that decreased levels of Wnt 1 signaling results in the downregulation of Ccnd1 that leads to apoptosis in several animal models. ${ }^{48-50}$ It is important to note that in mammals, there is partial compensation by the upregulation of $\mathrm{Ccnd} 2,{ }^{27}$ as it was observed in our cell system. It is also known that GSK3 $\beta$ overexpression induces increased apoptosis in vivo perturbing proliferation and maturation, resulting in the loss of immature neurons. ${ }^{51}$ The reduction of Wnt ligand levels or the removal of the canonical pathway component beta-catenin results in fewer neuroepithelial/radial glial stem cells and in precocious neuronal differentiation in mouse embryos. ${ }^{52}$

Further, it has been shown that in murine models of autism-like disorders, the most conspicuous phenotype is an increase in programmed cell death in the developing cerebellum. ${ }^{53,54}$ In CdLS patients, alterations in the development of cerebellar structures at anatomical levels are known, but more robust analyses with magnetic resonance imaging could help unraveling the link between autism features, seizures, and altered CNS development in these patients.

Increased apoptosis in our in vivo system led us to hypothesize that, at least to some extent, the mental retardation and autism-like behavior typically associated with CdLS patients could have their roots in this abnormality. The reported autopsy ${ }^{47}$ showed reduction of Purkinje cells number in the cerebellum, and our data could implicate an imbalance of cell death control during development as causative.

Several pieces of evidence suggest a possible regulation of the Wnt pathway by cohesins in non- dividing cells in different models. In skin fibroblasts expressing a mutated form of HDAC8, WNT5A, WNT2, WISP2, and FZD8 were found to be downregulated compared with wild-type cells. ${ }^{6}$ In Drosophila, SMC3 appears important to control expression of Flamingo/ 
Starry night (Fmi/Stan) the orthologs of vertebrate Celsr, which is known to transduce the Wnt signaling cascade in the non-canonical pathway to control cell polarity. ${ }^{55}$ It is interesting to note that this regulation might be two-way, as in colorectal cancer, the known upregulation of beta-catenin appears to control expression of SMC $3^{56}$

In conclusion, this paper shows for the first time, to our knowledge, a regulation of the Wnt-canonical pathway by cohesins during development in zebrafish embryos and in fibroblasts from CdLS patients. This suggests a potential underlying mechanism for the developmental abnormalities observed in the CNS of CdLS patients.

\section{Materials and Methods}

Animals. Breeding wild-type fish of the $A B$ strain were maintained at $28^{\circ} \mathrm{C}$ on a 14-h light/10-h dark cycle. Embryos were collected by natural spawning, staged according to Kimmel et al., ${ }^{57}$ and raised at $28^{\circ} \mathrm{C}$ in fish water (Instant Ocean, United Pet Group, Blacksburg, VA, USA; $0.1 \%$ methylene blue) in Petri dishes, according to established techniques, approved by the veterinarian (OVSAC) and the animal use committee (IACUC) at the University of Oregon, in agreement with local and national sanitary regulations. Embryonic ages are expressed in somites (s), or hours post fertilization (hpf).

To activate Wnt/beta-catenin signaling, LiCl was added to fish water for $30 \mathrm{~min}$ at 10-12 s stage at a concentration of $0.3 \mathrm{M}$ at $28^{\circ} \mathrm{C}$. Treated embryos were then washed three times with water and allowed to develop to $24 \mathrm{hpf}$.

In situ hybridization, histological analysis, and immunohistochemistry. WISH experiments were carried out as described by Thisse et al..$^{58}$ Immunohistochemistry analyses were performed according to the method described by Panzer et al. ${ }^{59}$ For each experiment, a minimum of 30 controls and MO-injected embryos were analyzed. To clone nipblb probe, a PCR was used (primers in Supplementary Table S1); and krox20 probe was synthesized as described in Oxtoby and Jowett; ${ }^{15}$ wnt1 probe was synthesized as described in Molven et al::22 axin2 probe was synthesized as described in Shimizu et al.:,55 atoh1 probe was synthesized as described in Millimaki et al.," ${ }^{23}$ pax2a probe was synthesized as described in Krauss et al.:21 hoxb2a probe was synthesized as described in Prince and colleagues. $^{20}$ For histological sections, stained embryos were re-fixed in $4 \%$ paraformaldehyde, dehydrated and stored in methanol, and wax-embedded and sectioned $(5 \mu \mathrm{m})$. For immunohistochemistry experiments, embryos were exposed to specific primary antibodies (Supplementary Table S3), followed by appropriate biotinylated secondary antibodies (Vector Laboratories, Burlingame, CA, USA). Images of embryos and sections were acquired using a microscope equipped with digital camera with LAS Leica Imaging software (Leica, Wetzlar, Germany). Images were processed using the Adobe Photoshop software (Adobe System Incorporated, San Josè, CA, USA), and, when necessary, different focal planes of the same image have been taken separately and later merged in a single image.

TUNEL staining. For TUNEL assay, a minimum of 24 embryos (per experimental group) were fixed with $4 \%$ paraformaldehyde for $2 \mathrm{~h}$ at room temperature. Embryos were washed with methanol at $-20^{\circ} \mathrm{C}$ and then twice with PBC $(0.001 \%$ Triton $X-100,0.1 \%$ sodium citrate in PBS) for 10 min. Staining for apoptotic cells was performed using the AP-In situ Cell Death Detection Kit (Roche Diagnostics, Penzberg, Germany) carefully leaving labeling reagents to react for the same length of time for all experiments. Embryos were incubated at $37^{\circ} \mathrm{C}$ for $1 \mathrm{~h}$, washed, stained, and mounted for microscopic imaging.

Injections. To repress nipblb mRNA translations, an ATG-targeting MO (nipblb-MO) was synthesized (Gene Tools LLC, Philomath, OR, USA), nipblb-MO: $5^{\prime}$-GTCCCCATTCATGCTGAAGAAGGGA-3', and used at the concentration of $1 \mathrm{pmol} / \mathrm{embryo}$ in $1 \times$ Danieau buffer $(\mathrm{pH} 7.6)$ as previously reported. ${ }^{60}$ As a control, a standard control MO oligonucleotide (ctrl-MO) was injected. In all experiments, nipblb-MO-injected embryos were compared with embryos injected with the same amount of ctrl-MO at the same developmental stage. p53-MO was designed (Gene Tools) and used as described in Robu et al. ${ }^{18}$

Cell cultures. Biopsies from four CdLS patients with known mutation in NIPBL and three healthy controls (Supplementary Table S4) were used in this study. Samples were obtained after signed informed consent. Healthy donors, two male and one female Italian pediatric patients, were undergoing surgical procedures for dermatological testing. Skin biopsies were collected from CdLS patients and pediatric controls, and then finely shredded using a scalpel in sterile conditions. Skin fragments were cultured in RPMI medium supplemented with $20 \%$ fetal bovine serum. After $\sim 7-10$ days, fibroblasts started to grow, and as soon as cell confluence was reached, cells were collected, following standard trypsinization procedure, suspended in Trizol reagent (Invitrogen, Life Technologies, Carlsbad, CA, USA) or radio immunoprecipitation assay buffer, and stored at $-80^{\circ} \mathrm{C}$. For any experiments, same passaged (maximum $P=3$ ) cells were compared.

Reverse transcription-PCR and real-time q-PCR assays. RNA extraction was performed following the manufacturer's protocol. Superscript II enzyme (Life Technologies) was used for cDNA synthesis. Quantitative-reverse transcription (RT)-PCRs on zebrafish embryos (minimum 30 per experimental group) were carried out in a total volume of $25 \mu$ l containing half volume of SYBR Green (Life Technologies). For normalization purposes, ef1-alpha RNA level was tested in parallel with the gene of interest. Primers are reported in Supplementary Table S1. Melting curve derivatives were assessed for each run.

q-RT-PCR experiments on human samples were performed with Universal Probe Master system (Roche Diagnostics). For this set of experiments, a Light Cycler 480II (Roche Diagnostics) was used.

Primers and probes were selected according to the Software Probe Finder (Roche Diagnostics) and are reported in Supplementary Table S2. ABL gene was used as reference and healthy patients cells as standard control.

Western blot. Fish embryos (minimum 30 per experimental group) and human fibroblasts were lysed in radio immunoprecipitation assay buffer and homogenized. Samples were boiled for $10 \mathrm{~min}$ at $95^{\circ} \mathrm{C}$ with $20 \times$ reducing agent (Bio-Rad, Hercules, CA, USA). Thirty micrograms of protein samples were size-fractionated by pre-casted gel (4-15\%, Bio-Rad) and transferred with following standard protocol. The polyvinylidene fluoride membranes (Bio-Rad) were blocked with $10 \%$ nonfat dry milk in TBST (TBS, pH 7.5, containing $0.1 \%$ Tween 20) for $1 \mathrm{~h}$ at room temperature and subsequently incubated with the primary antibody. Primary antibodies were used at appropriate working dilutions (Supplementary Table S3), in 5\% milkTBST over night at $4{ }^{\circ} \mathrm{C}$. Horseradish peroxidase-conjugated secondary antibodies (Sigma-Aldrich, St Louis, MO, USA) were used for $1 \mathrm{~h}$ at room temperature. The antigen signal was detected with the immune-star chemiluminescence detection system (Bio-Rad) as specified by the manufacturer. A StripAblot Stripping Buffer (Euroclone S.p.A., Milan, Italy) was used to recover membranes. Densitometry analyses were performed using Alliance instrument and Uviband software (Uvitec, Cambridge, UK). Beta-actin was used as reference for protein concentrations and then data was expressed normalized against control levels. For zebrafish embryos, experiments were run as three independent experiments and for human fibroblasts in duplicates.

Statistical analysis. For q-RT-PCR experiments, data were statistically analyzed applying a two-tailed $t$-test setting $P \leq 0.05$ as significant. Data were analyzed using the comparative $\Delta \Delta \mathrm{Ct}$ method ${ }^{20,60}$ both $t$-test and S.D. values refer to samples triplicates. For western blot experiments, raw data were analyzed applying a two-tailed paired $t$-test. Significance was set as $P<0.05$.

\section{Conflict of Interest}

The authors declare no conflict of interest.

Acknowledgements. We are grateful to the Italian National Association of Volunteers Cornelia de Lange for support and inspiration, and to all patients for consenting use of their tissues for this study. We are thankful to Mr. Eugenio Gautiero (Genetic Laboratory of San Gerardo Hospital, Monza, Italy) for technical advice in fibroblast isolation and culture and to Dr. Laura Corti and Prof. Emilio Berti for assisting in healthy donor samples recruitment. We would also like to express our deep gratitude to Prof. Finnell, Dr. Greene, Prof. Bellipanni and Dr. Gaston-Massuet for commenting the manuscript. This work was funded by Fondazione MBBM (Monza, Italy).

1. Kline AD, Krantz ID, Sommer A, Kliewer M, Jackson LG, FitzPatrick DR et al. Cornelia de Lange syndrome: clinical review, diagnostic and scoring systems, and anticipatory guidance. Am J Med Genet A 2007; 143A: 1287-1296.

2. Krantz ID, McCallum J, DeScipio C, Kaur M, Gillis LA, Yaeger D et al. Cornelia de Lange syndrome is caused by mutations in NIPBL, the human homolog of Drosophila melanogaster Nipped-B. Nat Genet 2004; 36: 631-635. 
3. Deardorff MA, Wilde JJ, Albrecht M, Dickinson E, Tennstedt S, Braunholz D et al. RAD21 mutations cause a human cohesinopathy. Am J Hum Genet 2012; 90: 1014-1027.

4. Lumsden A, Krumlauf R. Patterning the vertebrate neuraxis. Science 1996; 274: 1109-1115.

5. Altmann CR, Brivanlou AH. Neural patterning in the vertebrate embryo. Int Rev Cytol 2001; 203: $447-482$.

6. Deardorff MA, Bando M, Nakato R, Watrin E, Itoh T, Minamino M et al. HDAC8 mutations in Cornelia de Lange syndrome affect the cohesin acetylation cycle. Nature 2012; 489: 313-317.

7. Bose T, Gerton JL. Cohesinopathies, gene expression, and chromatin organization. J Cell Biol 2010; 189: 201-210

8. Dorsett $D$, Strom $L$. The ancient and evolving roles of cohesin in gene expression and DNA repair. Curr Biol 2012; 22: R240-R250.

9. Rollins RA, Morcillo P, Dorsett D. Nipped-B, a Drosophila homologue of chromosomal adherins, participates in activation by remote enhancers in the cut and Ultrabithorax genes. Genetics 1999; 152: 577-593.

10. Horsfield JA, Anagnostou SH, Hu JK, Cho KH, Geisler R, Lieschke G et al. Cohesindependent regulation of Runx genes. Development 2007; 134: 2639-2649.

11. Dorsett $D$. Running rings around chromosomes to trim axons and target dendrites. Dev Cell 2008; 14: 156-158.

12. Monnich M, Banks S, Eccles M, Dickinson E, Horsfield J. Expression of cohesin and condensin genes during zebrafish development supports a non-proliferative role for cohesin. Gene Expr Patterns 2009; 9: 586-594.

13. Kawauchi S, Calof AL, Santos R, Lopez-Burks ME, Young CM, Hoang MP et al. Multiple organ system defects and transcriptional dysregulation in the Nipbl $(+/-)$ mouse, a model of Cornelia de Lange Syndrome. PLoS Genet 2009; 5: e1000650.

14. Muto $A$, Calof $A L$, Lander AD, Schilling TF. Multifactorial origins of heart and gut defects in nipbldeficient zebrafish, a model of Cornelia de Lange Syndrome. PLoS Biol 2011; 9: e1001181.

15. Oxtoby $\mathrm{E}$, Jowett $\mathrm{T}$. Cloning of the zebrafish krox-20 gene (krx-20) and its expression during hindbrain development. Nucleic Acids Res 1993; 21: 1087-1095.

16. Rhodes JM, Bentley FK, Print CG, Dorsett D, Misulovin Z, Dickinson EJ et al. Positive regulation of c-Myc by cohesin is direct, and evolutionarily conserved. Dev Biol 2010; 344: 637-649.

17. Ghiselli G. SMC3 knockdown triggers genomic instability and p53-dependent apoptosis in human and zebrafish cells. Mol Cancer 2006; 5: 52.

18. Robu ME, Larson JD, Nasevicius A, Beiraghi S, Brenner C, Farber SA et al. p53 activation by knockdown technologies. PLoS Genet 2007; 3: e78.

19. Monnich M, Kuriger Z, Print CG, Horsfield JA. A zebrafish model of Roberts syndrome reveals that Esco2 depletion interferes with development by disrupting the cell cycle. PLoS One 2011; 6: e20051.

20. Prince VE, Moens CB, Kimmel CB, Ho RK. Zebrafish hox genes: expression in the hindbrain region of wild-type and mutants of the segmentation gene, valentino. Development 1998; 125: 393-406.

21. Krauss S, Johansen $T$, Korzh V, Fjose A. Expression pattern of zebrafish pax genes suggests a role in early brain regionalization. Nature 1991; 353: 267-270.

22. Molven A, Njolstad PR, Fjose A. Genomic structure and restricted neural expression of the zebrafish wnt-1 (int-1) gene. EMBO J 1991; 10: 799-807.

23. Millimaki BB, Sweet EM, Dhason MS, Riley BB. Zebrafish atoh1 genes: classic proneura activity in the inner ear and regulation by Fgf and Notch. Development 2007; 134: 295-305

24. Roussa E, Krieglstein K. Induction and specification of midbrain dopaminergic cells: focus on SHH, FGF8, and TGF-beta. Cell Tissue Res 2004; 318: 23-33.

25. Shimizu T, Yamanaka Y, Ryu SL, Hashimoto H, Yabe T, Hirata T et al. Cooperative roles of Bozozok/Dharma and Nodal-related proteins in the formation of the dorsal organizer in zebrafish. Mech Dev 2000; 91: 293-303.

26. Wallingford JB, Habas R. The developmental biology of Dishevelled: an enigmatic protein governing cell fate and cell polarity. Development 2005; 132: 4421-4436.

27. Ciemerych MA, Kenney AM, Sicinska E, Kalaszczynska I, Bronson RT, Rowitch DH et al. Development of mice expressing a single D-type cyclin. Genes Dev 2002; 16: 3277-3289

28. Staal FJ, Noort MvM, Strous GJ, Clevers HC. Wht signals are transmitted through $\mathrm{N}$-terminally dephosphorylated beta-catenin. EMBO Rep 2002; 3: 63-68.

29. Joly JS, Joly C, Schulte-Merker S, Boulekbache H, Condamine H. The ventral and posterior expression of the zebrafish homeobox gene eve1 is perturbed in dorsalized and mutant embryos. Development 1993; 119: 1261-1275.

30. Russo S, Masciadri M, Gervasini C, Azzollini J, Cereda A, Zampino G et al. Intragenic and large NIPBL rearrangements revealed by MLPA in Cornelia de Lange patients. Eur J Hum Genet 2012; 20: 734-741.

31. Adolf B, Bellipanni G, Huber V, Bally-Cuif L. atoh1.2 and beta3.1 are two new bHLHencoding genes expressed in selective precursor cells of the zebrafish anterior hindbrain. Gene Expr Patterns 2004; 5: 35-41.

32. Koster RW, Fraser SE. Direct imaging of in vivo neuronal migration in the developing cerebellum Curr Biol 2001; 11: 1858-1863.

33. Kim AS, Lowenstein DH, Pleasure SJ. Wnt receptors and Wnt inhibitors are expressed in gradients in the developing telencephalon. Mech Dev 2001; 103: 167-172.
34. van de Water S, van de Wetering M, Joore J, Esseling J, Bink R, Clevers $\mathrm{H}$ et al. Ectopic Wnt signal determines the eyeless phenotype of zebrafish masterblind mutant. Development 2001; 128: 3877-3888.

35. Heisenberg CP, Houart C, Take-Uchi M, Rauch GJ, Young N, Coutinho P et al. A mutation in the Gsk3-binding domain of zebrafish Masterblind/Axin1 leads to a fate transformation of telencephalon and eyes to diencephalon. Genes Dev 2001; 15: 1427-1434.

36. Klein PS, Melton DA. A molecular mechanism for the effect of lithium on development. Proc Natl Acad Sci USA 1996; 93: 8455-8459.

37. Wu D, Pan W. GSK3: a multifaceted kinase in Wnt signaling. Trends Biochem Sci2010; 35: 161-168.

38. Lustig B, Jerchow B, Sachs M, Weiler S, Pietsch T, Karsten U et al. Negative feedback loop of Wnt signaling through upregulation of conductin/axin2 in colorectal and liver tumors. $\mathrm{Mol}$ Cell Biol 2002; 22: 1184-1193.

39. Buchman JJ, Durak O, Tsai LH. ASPM regulates Wnt signaling pathway activity in the developing brain. Genes Dev 2011; 25: 1909-1914.

40. Gaston-Massuet C, Andoniadou CL, Signore M, Sajedi E, Bird S, Turner JM et al. Genetic interaction between the homeobox transcription factors HESX1 and SIX3 is required for normal pituitary development. Dev Biol 2008; 324: 322-333.

41. Martinez-Barbera JP, Rodriguez TA, Beddington RS. The homeobox gene Hesx1 is required in the anterior neural ectoderm for normal forebrain formation. Dev Biol 2000; 223: 422-430.

42. Andoniadou CL, Signore M, Young RM, Gaston-Massuet C, Wilson SW, Fuchs E et al. HESX1- and TCF3-mediated repression of Wnt/beta-catenin targets is required for normal development of the anterior forebrain. Development 2011; 138: 4931-4942.

43. Kim CH, Oda T, Itoh M, Jiang D, Artinger KB, Chandrasekharappa SC et al. Repressor activity of Headless/Tct3 is essential for vertebrate head formation. Nature 2000; 407: 913-916.

44. Lekven AC, Buckles GR, Kostakis N, Moon RT. Wnt1 and wnt10b function redundantly at the zebrafish midbrain-hindbrain boundary. Dev Biol 2003; 254: 172-187.

45. Dattani MT, Martinez-Barbera JP, Thomas PQ, Brickman JM, Gupta R, Wales JK et al. HESX1: a novel gene implicated in a familial form of septo-optic dysplasia. Acta Paediatr Suppl 1999; 88: 49-54.

46. Gaston-Massuet C, Kelberman D, Dattani M, Martinez-Barbera JP. Absence of SIX3 mutations in patients with congenital hypopituitarism. Am J Med Genet A 2009; 149A: 2874-2876.

47. Hayashi M, Sakamoto K, Kurata K, Nagata J, Satoh J, Morimatsu Y. Septo-optic dysplasia with cerebellar hypoplasia in Comelia de Lange syndrome. Acta Neuropathol 1996; 92: 625-630.

48. Roue G, Pichereau V, Lincet H, Colomer D, Sola B. Cyclin D1 mediates resistance to apoptosis through upregulation of molecular chaperones and consequent redistribution of cell death regulators. Oncogene 2008; 27: 4909-4920.

49. Wei W, Chua MS, Grepper S, So SK. Blockade of Wnt-1 signaling leads to anti-tumor effects in hepatocellular carcinoma cells. Mol Cancer 2009; 8: 76.

50. He B, You L, Uematsu K, Xu Z, Lee AY, Matsangou M et al. A monoclonal antibody against Wnt-1 induces apoptosis in human cancer cells. Neoplasia 2004; 6: 7-14.

51. Gomez-Sintes R, Hernandez F, Lucas JJ, Avila J. GSK-3 mouse models to study neuronal apoptosis and neurodegeneration. Front Mol Neurosci 2011; 4: 45.

52. Doe CQ. Neural stem cells: balancing self-renewal with differentiation. Development 2008; 135: $1575-1587$.

53. Tsai PT, Hull C, Chu Y, Greene-Colozzi E, Sadowski AR, Leech JM et al. Autistic-like behaviour and cerebellar dysfunction in Purkinje cell Tsc1 mutant mice. Nature 2012; 488: 647-651.

54. Yochum CL, Bhattacharya P, Patti L, Mirochnitchenko O, Wagner GC. Animal model of autism using GSTM1 knockout mice and early post-natal sodium valproate treatment. Behav Brain Res 2010; 210: 202-210.

55. Mouri K, Horiuchi SY, Uemura T. Cohesin controls planar cell polarity by regulating the level of the seven-pass transmembrane cadherin Flamingo. Genes Cells 2012; 17: 509-524.

56. Ghiselli G, Coffee N, Munnery CE, Koratkar R, Siracusa LD. The cohesin SMC3 is a target the for beta-catenin/TCF4 transactivation pathway. J Biol Chem 2003; 278: 20259-20267.

57. Kimmel CB, Ballard WW, Kimmel SR, Ullmann B, Schilling TF. Stages of embryonic development of the zebrafish. Dev Dyn 1995; 203: 253-310.

58. Thisse C, Thisse B, Schilling TF, Postlethwait JH. Structure of the zebrafish snail1 gene and its expression in wild-type, spadetail and no tail mutant embryos. Development 1993; 119: 1203-1215.

59. Panzer JA, Gibbs SM, Dosch R, Wagner D, Mullins MC, Granato M et al. Neuromuscular synaptogenesis in wild-type and mutant zebrafish. Dev Biol 2005; 285: 340-357.

60. Nasevicius A, Ekker SC. Effective targeted gene 'knockdown' in zebrafish. Nat Genet2000; 26: 216-220.

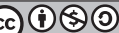

Cell Death and Disease is an open-access journal published by Nature Publishing Group. This work is licensed under a Creative Commons Attribution-NonCommercialShareAlike 3.0 Unported License. To view a copy of this license, visit http://creativecommons.org/licenses/by-nc-sa/3.0/ 